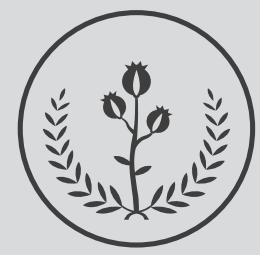

FUCS

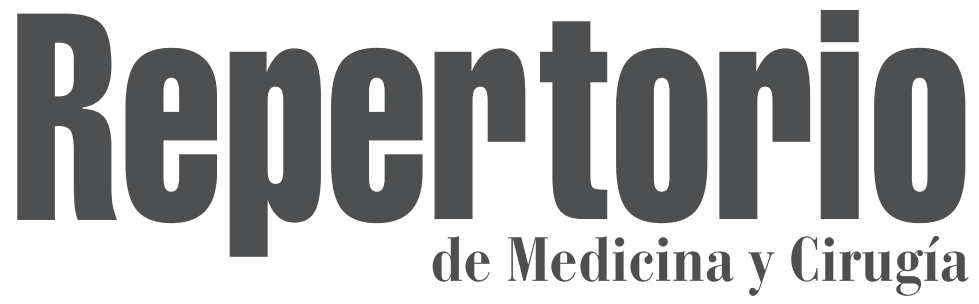

Vol. 30
№3. 2021

ISSN: 0121-7372 • ISSN electrónico: 2462-991X

Historía de la Medicina

\title{
Pneuma: receta colonial eficaz para el tratamiento de la disnea
}

\author{
Pneuma: effective colonial prescription to treat dyspnea
}

\begin{abstract}
Ignacio Briceño MD $^{\mathrm{a}}$
Eduardo Andrés Tuta ${ }^{\mathrm{b}}$

Sofía Barrera ${ }^{c}$

Julio César Martínez ${ }^{\mathrm{d}}$

Carlos Pacheco MDe
\end{abstract}

\footnotetext{
a Medicina - Genética Médica - PUJ // PhD Genética Molecular - Newcastle University, Universidad de Universidad de La Sabana. Bogotá DC, Colombia.

${ }^{b}$ Facultad de Medicina. Grupo Genética. Universidad de la Sabana. Bogotá DC, Colombia.

' Administración de Salud con énfasis / Especialización en Gerencia de Mercadeo, Grupo Genética Humana Universidad de La Sabana. Bogotá DC, Colombia.

${ }^{d}$ Facultad de Medicina Universidad de la Sabana. Bogotá DC, Colombia.

${ }^{e}$ Médico cirujano de la Universidad Libre de Barranquilla. Médico internista del Hospital Militar. Universidad Militar Nueva Granada. Cardiólogo de la Clínica Shaio. Universidad El Bosque. Bogotá DC, Colombia.
}

\section{R E S U M EN}

Objetivo: el archivo histórico Cipriano Rodríguez Santa María de la Universidad de La Sabana cuenta con múltiples recetas médicas empleadas por médicos y boticarios en el Nuevo Reino de Granada. Una de ellas prescribe el cacao para disminuir síntomas generados por afecciones del sistema respiratorio. Objetivos: describir el análisis de una receta médica empleada entre los siglos XVIII y XIX para el tratamiento del ahogo y describir la propiedad fitoterapéutica del cacao conocida gracias a la evidencia científica actual. Materiales y métodos: búsqueda documental en el Archivo Histórico Cipriano Rodríguez Santamaría de la Biblioteca Octavio Arizmendi Posada de la Universidad de La Sabana. Transcripción y análisis del documento denominado "Ahogo" y revisión de la literatura científica actual, así como de los textos originales, sin límite de tiempo. Resultados: la receta médica describe las características del cacao (Theobroma cacao) como agente fitoterapéutico con propiedades antiinflamatorias, analgésicas, antioxidantes e inmunomoduladoras aportadas por componentes como los ácidos oleico, esteárico, palmítico y flavonoides entre otros. La teobromina ejerce efectos broncodilatadores y antitusivos.

\section{INFORMACIÓN DEL ARTÍCULO}

Historia del artículo:

Fecha recibido: agosto 13 de 2020

Fecha aceptado: enero 21 de 2021
Autor para correspondencia:

Eduardo Andrés Tuta

eduardotuqu@unisabana.edu.co
DOI

10.31260/RepertMedCir.01217372.1088 
Conclusiones: el legado histórico colonial conservado en los archivos locales permite comprender racionalmente las propiedades de los agentes herbarios empleados para enfermedades y síntomas asociados. Existe evidencia científica que respalda el uso del cacao para disminuir la sintomatología asociada con el ahogo. Pese a ello, la escasa o nula descripción de la posología y los efectos secundarios, dificulta analizar la eficacia de esta tradición. En consecuencia, no se puede establecer su eficacia científicamente.

Palabras clave: disnea, recetas médicas, historia de la medicina, cacao.

(C) 2021 Fundación Universitaria de Ciencias de la Salud -FUCS. Este es un artículo Open Access bajo la licencia CC BY-NC-D (http:// creativecommons.org/licenses/by-nc-nd/4.0/)

\section{A BS TRACT}

Objetive: The Cipriano Rodríguez Santamaria Historical Archive at Universidad de La Sabana includes multiple medical prescriptions used by physicians and apothecaries in the New Kingdom of Granada. Among them, cocoa was prescribed to relieve symptoms generated by respiratory diseases. Objectives: to describe the analysis of a medical prescription used between the 18th and 19th centuries to treat shortness of breath ("ahogo") and to describe the phytotherapeutic properties of cocoa through current scientific evidence. Materials and Methods: a documentary search in the Cipriano Rodríguez Santamaria Historical Archive: Octavio Arizmendi Posada Library, Universidad de La Sabana. Transcription and analysis of the document named "ahogo" and review of the current scientific literature, as well as, of the original texts, with no time limit. Results: the medical prescription describes the characteristics of cocoa (Theobroma cacao) as a phytotherapeutic agent featuring anti-inflammatory, analgesic, antioxidant and immunomodulatory properties provided by components such as oleic, stearic, palmitic and flavonoid acids, among others. Theobromine exerts a bronchodilator and antitussive effect. Conclusions: the colonial historical legacy preserved in local archives allows a rational understanding of the properties of herbal agents as treatment for diseases and their symptoms. There is scientific evidence supporting the use of cocoa to reduce the symptoms associated with dyspnea. However, little or no description of dosage and side effects makes it difficult to analyze the efficacy of this tradition. Consequently, its efficacy cannot be scientifically established.

Keywords: dyspnea, medical prescriptions, history of medicine, cocoa.

(C) 2021 Fundación Universitaria de Ciencias de la Salud - FUCS. This is an open access article under the CC BY-NC-ND license (http://creativecommons.org/licenses/by-nc-nd/4.0/).

\section{INTRODUCCIÓN}

Parte de la medicina actual se forjó gracias a la evolución del conocimiento en anatomía y fisiología a lo largo de la historia. Entender el aparato respiratorio y sus patologías asociadas fueron atractivos enigmas para médicos $\mathrm{y}$ civilizaciones antiguas. En Grecia "pneuma" ( $\pi \vee \varepsilon \cup ̃ \mu \alpha)$ significaba "respiración", y esta se relacionaba con la armonía entre humores corporales y la espiritualidad. ${ }^{1}$ Antes de los estudios realizados por Galeno de Pérgamo (129-200 d.C.), la historia de la fisiología de la respiración y estructuras anatómicas asociadas se atribuyen a Platón (428399 a. C.) e Hipócrates de Cos (460-356 a.C.) entre otros., ${ }^{2,3}$ Pese al desconocimiento de conceptos indispensables para el entendimiento del cuerpo y sus patologías, la capacidad de observación y análisis de personas sanas y enfermas, permitieron invaluables descubrimientos.

El uso de sustancias medicinales de origen natural ha sido protagonista en la lucha contra múltiples enfermedades. Descubrimientos y prácticas durante el periodo colonial permitieron el desarrollo de recetas médicas para el tratamiento de enfermedades o síntomas asociados. El objetivo principal de este trabajo es contextualizar la formulación de una receta médica del siglo XVIII en el Virreinato de la Nueva Granada (actual Colombia), la cual contiene recomendaciones para el tratamiento del ahogo por medio de sustancias medicinales de origen natural.

\section{MATERIALES Y MÉTODOS}

Búsqueda documental en el archivo histórico de la Biblioteca Octavio Arizmendi Posada de la Universidad de La Sabana y análisis de la receta médica colonial «ahogo». Para este propósito se realizó una revisión de textos originales haciendo un recorrido histórico a través de la evolución del conocimiento de la respiración, sus múltiples definiciones y funciones. De manera simultánea se recopiló evidencia 
científica disponible sobre las sustancias fitoterapéuticas que componen la receta en estudio. Bases de datos como Scopus/ PubMed/ y Google Académico fueron utilizados sin límite de tiempo y teniendo en cuenta los siguientes términos de búsqueda: fitoterapia, extractos de plantas, uso terapéutico, agua y cacao (Theobroma cacao).

\section{RES ULTADOS}

\section{Respiración según las antiguas civilizaciones}

La consideración histórica de la función de la respiración era enfriar la sangre mientras era distribuida por todo el cuerpo por medio de las arterias. Hipócrates de Cos (460370 a.C.) enseñaba el examen físico del aparato respiratorio a través de la auscultación directa dentro del concepto griego de la medicina, el entendía el proceso respiratorio como sinónimo del alma. ${ }^{1}$ Años después se empezaron a considerar otros órganos como parte del proceso respiratorio; entonces el cerebro, el corazón y los pulmones entraron en debate sobre cuál era el responsable de la respiración, lo que originó diferentes posturas adoptadas en las escuelas de medicina antiguas. El pensamiento cerebrocentrista sostenía que el alma se originó del aire (pneuma) y que éste ingresaba al cuerpo a través del cerebro encargado de distribuirlo, por otro lado la postura cardiocentrista establecía que el corazón era el protagonista. ${ }^{2}$ Platón (428-347 a.C.), describió al corazón como el encargado del coraje y la respiración, esta última como ya lo había mencionado Hipócrates, enfriaba la sangre. ${ }^{2,3}$ Platón al igual que Empédocles (490430 a.C.), incorporaron el concepto de la utilidad de los poros de la piel en la mecánica respiratoria e intercambio gaseoso. Como un circuito el aire entraba por la nariz y la boca enfriándose, e inmediatamente era expulsado por los poros de la piel calentándose en el proceso. 1 Como discípulo de Platón, Aristóteles (384-322 a.C.) tenía una postura cardiocentrista y descartó cualquier utilidad del diafragma en la respiración. ${ }^{1-3}$

\section{Movimientos respiratorios y el diafragma}

Galeno de Pérgamo (129-200 d.C.) adoptó conocimientos

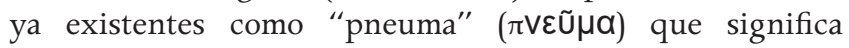
"respiración", pero descartó la existencia de aire en las arterias entre otros. ${ }^{4}$ Los primeros acercamientos al aparato respiratorio se hicieron al observar las lesiones en los gladiadores, consecuencia de sus temibles enfrentamientos. Su amplio conocimiento y capacidad de curar heridas de guerra le otorgó el nombramiento como médico del ejército romano de Marco Aurelio (121-180 d.C). ${ }^{5}$ Galeno desconocía la asociación entre la ausencia del aire y la muerte rápida para cualquier ser vivo. ${ }^{6}$ A pesar de que sus primeras disecciones estaban encaminadas a responder preguntas sobre la fisiología de la respiración, experimentos y descripciones detalladas en animales vivos permitieron conocer y entender las funciones de los músculos intercostales y el diafragma, además de sus respectivas inervaciones. Concluyó que dichos músculos eran los encargados de los movimientos del tórax y cualquier daño en los nervios imposibilitaba su extensión completa y por consiguiente afectaba los movimientos respiratorios. ${ }^{5}$

\section{Estado de las enfermedades en la Nueva Granada}

Con la distribución epidemiológica de las enfermedades antes de la llegada de los colonos al nuevo mundo, esta región era azotada por enfermedades como la tuberculosis, treponematosis (sífilis), tripanosomiasis, leishmaniasis (uta), fiebre oroya, verruga peruana y afecciones articulares (reumatismo y gota) entre otras. ${ }^{7}$ Pese a la ausencia de un tratamiento efectivo para dichas enfermedades, la población indígena empleaba agentes medicinales procedentes solo de plantas y/o animales. ${ }^{8}$

Durante el periodo colonial confluyeron una mezcla de conocimientos y prácticas médicas del nuevo y viejo mundo que sirvieron para controlar enfermedades ya existentes en América, así como la aparición de nuevas.

Un entorno con alto grado de insalubridad y epidemias arrasaron con los escasos médicos disponibles impidiendo el manejo de los enfermos. ${ }^{9}$ Pese a ello, las boticas médicas como parte del sistema de salud neogranadino, proporcionaban recetas médicas en su gran mayoría de origen vegetal para el manejo de la viruela y el sarampión entre otras enfermedades, así como de sus síntomas asociados. ${ }^{10,11}$

\section{Manejo del ahogo en el periodo neogranadino}

A continuación se realiza la transcripción de manera literal de la receta médica prescrita para el Ahogo: Haí reciente

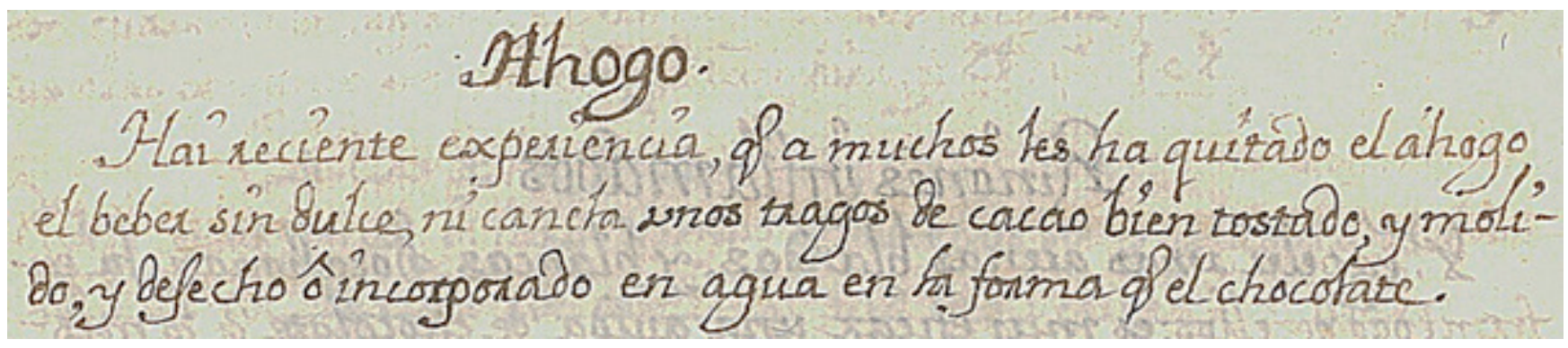

Figura 1. . Receta para ahogo. Referencia: Archivo Histórico Cipriano Rodríguez Santamaría. Biblioteca Octavio Arizmendi Posada, Universidad de La Sabana. Caja 10, Carpeta 2 (2 PDF) , Folios 82. Disponible en: https://intellectum.unisabana.edu.co/handle/10818/18140. 
experiencia, q a muchos les ha quitado el ahogo el beber sin dulce, ni canela, uno tragos de cacao bien tostado, y molido, y derecho o incorporado en agua en la forma que el chocolate (figura 1).

\section{Cacao en el manejo colonial del ahogo}

Cacao (Theobroma cacao): el árbol del cacao es una de las plantas más utilizadas a lo largo de la historia debido a sus características y propiedades benévolas. Los olmecas fueron los primeros en cultivar dicha planta en Mesoamérica dando origen al término cacao. ${ }^{12}$ Simultáneamente los mayas la nombraron "kakawa" que traduce "comida de los dioses". Solo cuando Cristóbal Colón pisó tierra americana y descubrió el cacao fue utilizado como medicina en diferentes latitudes de Europa. ${ }^{13}$ En la actualidad se conocen sus propiedades antiinflamatorias, analgésicas, antioxidantes e inmunomoduladoras debido en gran proporción a la manteca de cacao (ácido oleico, esteárico y palmítico), flavonoides, polifenoles y metilxantinas entre otros. ${ }^{14,15}$ Además, de ser fuente importante de vitamina $\mathrm{D}^{16}$ y teobromina. ${ }^{17}$ Esta última es inhibidora de los receptores de adenosina, por lo que ejerce un efecto estimulante sobre el sistema nervioso central e incrementa las concentraciones AMPc y GMPc. ${ }^{18}$ A partir de ello genera un efecto diurético al aumentar la filtración glomerular y la disminución de la reabsorción tubular, acción procinética, cronotrópica e inotrópica positiva, broncodilatadora y antitusiva. ${ }^{19-22}$

\section{Agua, elemento esencial para el hombre}

El agua es un nutriente esencial que interviene en diversos mecanismos fisiológicos y su distribución corporal responde a la necesidad de mantener la homeostasis hídrica. ${ }^{23}$ El uso del agua para el manejo de algunas enfermedades es uno de los procedimientos más descritos a través de la historia desde los orígenes de la humanidad. ${ }^{24}$ En la actualidad se conoce por ser el disolvente universal para compuestos iónicos y múltiples solutos como son la glucosa y los aminoácidos.

Es esencial para mantener homeostasis celular porque ayuda a transportar nutrientes hacia las células y moviliza el desecho de las mismas. Funciona como termorregulador ya que al tener un alto calor específico y un alto calor de vaporización contribuye a limitar los cambios en la temperatura corporal en un ambiente cálido o frío. Además funciona como lubricante para todos los sistemas, articulaciones, mucosa gástrica e intestinal, secreción de moco en el sistema respiratorio y genitourinario. ${ }^{25,26}$

\section{Alimentos o medicamentos, amplia discusión de la receta colonial}

Las plantas han sido fuente de alimentos y medicinas para el ser humano mejorando su calidad de vida desde el inicio de los tiempos. Mucho antes del arribo de los conquistadores a América, los amerindios eran poseedores de una diversidad gastronómica que fue extendida por costumbres europeas desde el momento de la conquista. ${ }^{27}$ Con el pasar del tiempo, culturas y prácticas médicas del viejo continente enriquecieron el manejo de las enfermedades en América a partir de medicinas de origen animal vegetal o mineral. En el siglo XVIII boticarios españoles con amplia experiencia crearon manuales terapéuticos para la formación de sus aprendices en el nuevo mundo. Influenciados por conocimientos y prácticas médicas españolas y árabes, estos manuales explican tratamientos con base en especial de agentes de origen vegetal. Las limitaciones en la obtención o producción de ciertas plantas requirieron que se diera la importación de materia prima desde Europa, elevando sus costos e impidiendo en cierta medida el acceso a la totalidad de la población neogranadina. Sin embargo, la Expedición Botánica liderada por José Celestino Mutis, permitió descubrir fauna y flora indispensables para el desarrollo de nuevos remedios y suplir la ausencia de medicinas en

\section{CONCLUSIONES}

Durante el periodo colonial el manejo de las enfermedades se basaba en el conocimiento de plantas y sustancias derivadas de estas. Tras una breve revisión de la literatura científica actual acerca del cacao como principal agente utilizado en la receta colonial para el manejo del "ahogo", encontramos propiedades broncodilatadoras y antitusivas, las cuales explicarían la mejoría de los síntomas del "ahogo". Además, las otras propiedades asociadas con el cacao como las antiinflamatorias, analgésicas, antioxidantes e inmunomoduladoras pudiesen actuar para controlar manifestaciones como la disnea y demás síntomas respiratorios. Estos hallazgos permiten entender el uso generalizado en esa época de este tratamiento basado en el cacao para el manejo de las complicaciones respiratorias. Si bien la falta de información relacionada con la posología $\mathrm{y}$ efectos secundarios no permite sustentar de manera científica su uso, resulta meritorio que con los escasos recursos de la época lograran establecer un tratamiento que en buena medida aliviaba la sintomatología de los pacientes afectados. Existe evidencia científica que respalda el uso del cacao para disminuir la sintomatología asociada con el ahogo.

\section{REFERENCIAS}

1. Derenne JP, Debru A, Grassino AE, Whitelaw WA. The earliest history of diaphragm physiology. Eur Respir J. 1994;7(12):22342240. doi: 10.1183/09031936.94.07122234

2. Fitting JW. From breathing to respiration. Respiration. 2015;89(1):82-7. doi: 10.1159/000369474.

3. French RK: The thorax in history 1. From ancient times to Aristotle. Thorax 1978;33:10-18.

4. Siegel RE. Galen's System of Physiology and Medicine. Basel, S. Karger, 1968. 
5. Derenne JP, Debru A, Grassino AE, Whitelaw WA. History of diaphragm physiology: the achievements of Galen. Eur Respir J. 1995;8(1):154160. doi:10.1183/09031936.95.08010154.

6. Furley DJ, Wilkie JS. Galen on respiration and the arteries. Princeton, Princeton University Press, 1989.

7. Rodríguez Cuenca, J.V. Las enfermedades en las condiciones de vida prehispánica de Colombia. la Ed. Bogotá. Guadalupe. 2006.

8. Guzman Urrego, M.A. Sífilis diagnóstico y manejo serológico. 3a Ed. Bogotá. Instituto Nacional de Salud. 1983.

9. Pico RP. Las boticas en el Nuevo Reino de Granada a finales del período colonial: El lento camino hacia la modernidad. 2015;37(3):223-41. Available from: revistamedicina.net/ojsanm/ index.php/Medicina/article/view/110-3/836.

10. Lozano A, Martínez JC, Uribe J, Gómez A, Figueredo SA, Briceño I. De curandis hominum morbis: Una receta médica del siglo XVIII en el Nuevo Reino de Granada para el sarampión y las viruelas. Biomédica. 2020;40(3):427-437. doi: 10.7705/biomedica.4995.

11. Uribe-Vergara Jorge, Briceño-balcazar Ignacio, Martínez-Lozano Julio, Pacheco-Cuentas Carlos, Tuta-Quintero Eduardo, RuedaRodríguez Alejandro, Gómez-Gutiérrez Alberto. Formulación para reducir las palpitaciones cardíacas, una receta médica de mediados del siglo XVIII en el Nuevo Reino de Granada. Arch Cardiol Mex. 2020;90(2): 138-143. doi: 10.24875/ACM.19000268.

12. Kaufman T, Justeson J. The history of the word for cacao in ancient mesoamerica. Ancient Mesoamerica. 2007;18(2):193-237. doi: 10.1017/S0956536107000211.

13. Pucciarelli DL, Grivetti LE. The Medicinal Use of Chocolate in Early North America. Mol. Nutr. Food Res. 200852:1215-1227. doi: 10.1002/mnfr.200700264.

14. Rusconi M, Conti A. Theobroma cacao L., the Food of the Gods: a scientific approach beyond myths and claims. Pharmacol Res. 2010;61(1):513. doi:10.1016/j.phrs.2009.08.008.

15. De Araujo QR, Gattward JN, Almoosawi S, Silva Md, Dantas PA, De Araujo Júnior QR. Cocoa and Human Health: From Head to Foot--A Review. Crit Rev Food Sci Nutr. 2016;56(1):112. doi:10.10 80/10408398.2012.657921.
16. Kühn J, Schröter A, Hartmann BM, Stangl GI. Cocoa and chocolate are sources of vitamin D2. Food Chem. 2018;269:318320. doi: 10.1016/j.foodchem.2018.06.098.

17. Ellam S, Williamson G. Cocoa and human health. Annu Rev Nutr. 2013;33:105128. doi: 10.1146/annurev-nutr-071811-150642.

18. Smit HJ. Theobromine and the pharmacology of cocoa. Handb Exp Pharmacol. 2011;(200):201234. doi: 10.1007/978-3-642-13443-2_7.

19. Kerimi A, Williamson G. The cardiovascular benefits of dark chocolate. Vascul Pharmacol. 2015;71:1115. doi: 10.1016/j.vph.2015.05.011.

20. Engler $M B$, Engler $M M$, Chen $C Y$, et al. Flavonoid-rich dark chocolate improves endothelial function and increases plasma epicatechin concentrations in healthy adults. J Am Coll Nutr. 2004;23(3):197204. doi: 10.1080/07315724.2004.10719361.

21. Langer S, Marshall LJ, Day AJ, Morgan MR. Flavanols and methylxanthines in commercially available dark chocolate: a study of the correlation with nonfat cocoa solids. J Agric Food Chem. 2011;59(15):84358441. doi: 10.1021/jf201398t.

22. Usmani O, Belvisi M, Patel H,Crispino N, Birrell M,Korbonits M, Korbonits D, Barnes J.Theobromine inhibits sensory nerve activation and cough. The FASEB Journal. 2005;19(2):231-233. doi: 10.1096/fj.04-1990fje

23. Rush EC. Water: neglected, unappreciated and under researched. Eur J Clin Nutr. 2013;67(5):492495. doi: 10.1038/ejcn.2013.11.

24. Pérez-Fernández M.R, Novoa-Castro B. Historia del agua como agente terapéutico. Fisioterapia. 2002;24(52):3-13. doi: 10.1016/ S0211-5638(01)73022-2

25. Jéquier E, Constant F. Water as an essential nutrient: the physiological basis of hydration. Eur J Clin Nutr. 2010;64(2):115123. doi: 10.1038/ejcn.2009.111.

26. Sawka MN, Cheuvront SN, Carter R 3rd. Human water needs. Nutr Rev. 2005;63(6 Pt 2):S30-S39. doi: 10.1111/j.1753-4887.2005. tb00152.x.

27. Saldarriaga, Gregorio. Alimentación e identidades en el Nuevo Reino de Granada siglos XVI y XVII. 2a Ed. Bogotá: Ministerio de Cultura. 2012. 